\title{
Coronary heart disease epidemiology: a look towards the South
}

In this issue of the journal, Masiá and coauthors ${ }^{1}$ assess cardiovascular risk factors (CRF) in a Spanish province where coronary heart disease (CHD) incidence is very low when compared with incidence rates observed in northern Europe and North America. As very few data on cardiovascular epidemiology in southern Europe are available, this work is timely. Besides the results reported by Masiá et al we await the final results of the MONICA Project. $^{2}$ The comparison between the results provided by the MONICA Projects in Spain, France, and Italy and the results presented by Masiá et al will be interesting.

In brief, they conclude that despite low CHD incidence, CRF prevalence was relatively high. ${ }^{1}$ This conclusion is provocative. Indeed, epidemiology has identified major risk factors and randomised trials have shown interest in early primary and secondary CHD prevention. Hypercholesterolaemia treatment in primary ${ }^{3}$ and secondary ${ }^{4}$ prevention lengthens life expectancy and decreases the number of CHD events. Preventive cardiology is now part of public health and should have the same status in medical studies as curative medicine.

From a methodological point of view, CRF measurements remain problematic because only one measurement is generally performed in cross sectional studies. Single examinations of CRF cannot do more than act as a crude proxy for a lifetime exposure of varying levels of these CRF. Repeated measures over a lifetime should be more appropriate. This is true for most of the cross sectional surveys. The second problem is the measurement standardisation, and Masiá et al have taken great care in the CRF measurements and have profited from the Quality Assessment of the WHO MONICA Project. It is therefore highly improbable that measurement errors could account for the results presented.

As discussed by the authors, CHD incidence observed today is the true indication of exposure to CRF in the past. In other words, high CRF levels recorded today in Spain may account for high CHD incidence in the future. This may be the case in Gerona where tobacco consumption is $45.6 \%$ in women aged $25-34$ years. The REGICOR Registry will provide some answers in a few years. In contrast, it would be interesting to have data on CRF levels in Gerona a few years ago corroborating CHD incidence observed today by the REGICOR investigators.

This reflection on CRF and CHD incidence is in agreement with the reflection on multiple risk function for predicting $\mathrm{CHD}$. It is almost certain that major risk factors (cigarette smoking, high cholesterol, high blood pressure, and diabetes) are strong CHD predictors in southern Europe as well. In a study comparing four cohort studies, (Paris prospective study, European and American cohorts of the Seven Countries Study and the Pooling Project) it was demonstrated that major risk factor coefficients in multivariate analyses were similar. ${ }^{5}$ In a cohort study, still in progress (PRIME Study), major risk factors are CHD predictors in a prevalence case-control study performed at entry in France and in Northern Ireland, two countries with very different CHD incidence. ${ }^{6}$ In a study comparing Toulouse (south western France) and Belfast (Northern Ireland), we have tried to explain differences in CHD death rates observed between the two countries (ratio of rates Belfast compared with Toulouse: $4.2: 1)$ through the analysis of risk factors. ${ }^{7}$ Using two multiple logistic functions, the probability of developing CHD over the next four to five years was similar in Toulouse and in Belfast. In other words, main risk factors cannot account for all the differences of CHD risk observed between southern France and Northern Ireland.
This apparent paradox, high CRF and low CHD incidence, must be studied in the light of a relative knowledge of CHD genesis. Indeed, coronary atheroma lesions seem identical from the north to the south of Europe but with lower CHD incidence in the south. In the studies available for southern Europe, ${ }^{15-7}$ results seem to show that $\mathrm{CRF}$ are close to those observed in northern Europe. How can we then explain the differences found in CHD incidence?

In the first place, epidemiology should get involved in the analysis of CRF that are not usually extensively studied. This is the case for physical exercise, dietary habits, and alcohol consumption. In particular, red wine, rich in polyphenols, favours the decrease of HDL-cholesterol and inhibits LDL oxidation ${ }^{8}$ and therefore could account for the relative protection of southern Europe against CHD. Environmental factors should be studied on a population scale. Similarly, the analysis of the various lifestyles, particularly at work, ${ }^{9}$ is promising.

This paradox of the Spanish results ${ }^{1}$ could be related to the "French Paradox" described a few years ago. ${ }^{10}$ These two paradoxes are nothing more than a mere confirmation of what has already been demonstrated several times: CRF increase CHD risk. But they become very useful when they focus on protective factors. We have already mentioned physical exercise, dietary habits, and alcohol consumption. The present theory on atherosclerosis ${ }^{11}$ and the role of social factors ${ }^{9}$ in the occurrence of the disease deserve more specific studies. After several years of intensive screening of countries with high CHD incidence it would be wise to study countries with low incidence and to promote protective CRF. From a public health point of view the fight against risk factors must go on. For instance, diets poor in saturated fats and salt should be favoured but we must not forget to analyse genes, environmental factors, and gene/environment interactions responsible for low CHD incidence. A preponderant position of protective interactions in public health seems to be fundamental too.

JEAN FERRIÈRES

Department of Epidemiology, Faculty of Medicine, 37 allees fules Guesde 31073 Toulouse cedex, France

1 Masiá R, Pena A, Marrugat J, et al, and the REGICOR Investigators. High prevalence of cardiovascular risk factors in Gerona, Spain, a province with low myocardial infarction incidence. If Epidemiol Community Health 1998;52:707-15

2 Tunstall-Pedoe $\mathrm{H}$, Kuulasmaa $\mathrm{K}$, Amouyel $\mathrm{P}$, et al. Myocardial infarction and coronary deaths in the World Health Organization MONICA Project. Registration procedures, event rates, and case-fatality rates in 38 populations from 21 countries in four continents. WHO MONICA Project. Circulation 1994;90:583-612.

3 Shepherd J, Cobbe SM, Ford I, et al. Prevention of coronary heart disease with pravastatin in men with hypercholesterolemia. $N$ Engl $f$ Med 1995;333:1301-6.

4 Scandinavian Simvastatin Survival Study Group. Randomised trial of cholesterol lowering in 4444 patients with coronary heart disease: the

Scandinavian Simvastatin Survival Study (4S). Lancet 1994;344:1383-9. Ducimetiere P, Richard JL, Cambien F, et al. Coronary heart disease in
middle-aged frenchmen. Comparisons between Paris Prospective Study, middle-aged frenchmen. Comparisons between Paris Prospective Stud

6 Ferrières J, Evans A, Amouyel P, et al. Risk factors in two populations at contrasting risk for coronary artery disease. J Am Coll Cardiol 1998;31 (suppl A): $147 \mathrm{~A}$.

7 Evans A, Ruidavets JB, McCrum E, et al. Autres pays, autres coeurs ? Dietary patterns, risk factors and ischaemic heart disease in Belfast and Toulouse. Q F Med 1995;88:469-77.

8 Frankel EN, Kanner J, German JB, et al. Inhibition of oxidation of human low-density lipoprotein by phenolic substances in red wine. Lancet 1993,341:454-7.

9 Marmot MG, Bosma H, Hemingway H, et al. Contribution of job control and other risk factors to social variations in coronary heart disease incidence. Lancet 1997;350:235-9.

10 Richard JL, Cambien F, Ducimetière P. Particularités épidémiologiques de la maladie coronarienne en France. Presse Med 1981;10:1111-14.

11 Berliner JA, Navab M, Fogelman AM, et al. Atherosclerosis: basic mechanisms: oxidation, inflammation, and genetics. Circulation 1995;91: 2488-96. 